S. Turner

Nagoya Math. J.

Vol. 77 (1980), 1-4

\title{
PRINCIPAL POLARIZATIONS OF ABELIAN SURFACES OVER FINITE FIELDS
}

\author{
STUART TURNER
}

In $\S 1$ of this note we construct abelian varieties of dimension two defined over $\boldsymbol{F}_{p^{n}}, n$ odd, which admit infinitely many distinct principal polarizations. These polarizations determine an infinite family of geometrically non-isomorphic complete singular curves defined and irreducible over $\boldsymbol{F}_{p^{n}}$ which have isomorphic Jacobian varieties. In $\S 2$ we calculate the zeta function of these curves.

$\S 1$.

Let $q=p^{n}, n$ odd, and $\pi=q^{1 / 2}$. Then $\{ \pm \pi\}$ is a conjugacy class of Weil numbers for $q$ which corresponds to an isogeny class of simple abelian varieties defined over $F_{q}([1],[5])$. Let $A$ be a member of this isogeny class, End $(A)$ be the ring of $\boldsymbol{F}_{q}$-endomorphisms of $A$ and $E=\operatorname{End}(A) \otimes \boldsymbol{Q} . \quad E$ is a division algebra with center $\Phi=\boldsymbol{Q}(\pi) . \quad E$ has invariant $1 / 2$ at each of the real places of $\Phi$, invariant zero at each finite place of $\Phi$ prime to $p$, and invariant

$$
\operatorname{ord}_{v}(\pi) \cdot\left[\Phi_{v}: \boldsymbol{Q}_{p}\right] / \operatorname{ord}_{v}(q) \quad(\bmod 1)
$$

at the one place $v$ of $\Phi$ which lies over $p$; $v$ is ramified so this last invariant is zero. Finally, $2 \operatorname{dim} A=[E: \Phi]^{1 / 2}[\Phi: Q]$, ([5], [6], [8]). Since $[E: \Phi]^{1 / 2}$ is the least common multiple of the denominators of the invariants, $\operatorname{dim} A=2$.

End $(A)$ contains $\pi([7]$, Prop. 3.5$)$, so $Z\left[p^{1 / 2}\right] \subset$ End $(A) . \quad Z\left[p^{1 / 2}\right]$ is an order in $\Phi$ (the maximal order if $p \equiv 2,3(\bmod 4))$, so by Dirichlet's theorem, the units in $Z\left[p^{1 / 2}\right]$ form a group isomorphic to $\{ \pm 1\} \times Z$. Hence Aut $A$, the group of $F_{q}$-automorphisms of $A$, contains an element of infinite order.

$A \times \operatorname{Spec} \boldsymbol{F}_{q^{2}}$ has Weil number $\pi^{2}=q$. Using the theorem of Tate cited above one sees that the isogeny class of simple abelian varieties

Received February 24, 1978. 
defined over $\boldsymbol{F}_{q^{2}}$ with Weil number $q$ consists of elliptic curves. Since their endomorphism algebra is a quaternion algebra over $\boldsymbol{Q}$ with invariants $1 / 2$ at the real place of $\boldsymbol{Q}$ and at the $p$-adic place, they are supersingular curves ([4], p. 217). So $A \times \operatorname{Spec} \boldsymbol{F}_{q^{2}}$ is $\boldsymbol{F}_{q^{2}}$-isogenous to a product of isomorphic supersingular elliptic curves. Let $E$ be a curve in this isogeny class. The Frobenius automorphism $F \in \operatorname{Gal}\left(\boldsymbol{F}_{q^{2}} / \boldsymbol{F}_{q}\right)$ transforms $E$ into an elliptic curve $E^{(q)}$ and there is a canonical purely inseparable isogeny $i: E \rightarrow E^{(q)} . \quad E \times E^{(q)}$ is $F_{q^{2}}$-isogenous to $A \times \operatorname{Spec} \boldsymbol{F}_{q^{2}}$.

Let 0 (resp. $0^{\prime}$ ) be a rational point on $E$ (resp. $E^{(q)}$ ). Define a group law on $E$ (resp. $E^{(q)}$ ) such that 0 (resp. $0^{\prime}$ ) is the identity for this addition. Let $X=E \times\left\{0^{\prime}\right\}+\{0\} \times E^{(q)}$. $X$ is a divisor on $E \times E^{(q)}$, rational over $\boldsymbol{F}_{q^{2}}$. $X$ determines a principal polarization $\mathscr{C}(X)$ on $E \times E^{(q)}$.

Proposition. $F_{q}$ is a field of definition for the principally polarized abelian variety $\left(E \times E^{(q)}, \mathscr{C}(X)\right)$.

Proof. The proposition asserts the existence of an abelian variety $B$ defined over $\boldsymbol{F}_{q}$, of a $\boldsymbol{F}_{q}$-rational divisor $Y$ on $B$, and of a $\boldsymbol{F}_{q^{2}}$-isomorphism $\psi: B \times \operatorname{Spec} F_{q^{2}} \rightarrow E \times E^{(q)}$ such that $\psi\left(Y \times F_{q^{2}}\right)=X$. To show that this. descent is possible it suffices to construct a cocycle $h \in Z^{1}\left(G\right.$, Aut $\left.\left(E \times E^{(q)}\right)\right)$ and an $F_{q}$-isomorphism $\phi: h_{F}\left(E \times E^{(q)}\right) \rightarrow\left(E \times E^{(q)}\right)^{F}$ such that $\phi\left(h_{F}(X)\right)$ $=X^{F}$.

Let $e$ be the identity of $G$ and $h_{e}$ be identity automorphism of $E \times E^{(q)}$. Let $h_{F}: E \times E^{(q)} \rightarrow E^{(q)} \times E$ be the automorphism which interchanges the factors of the product. One verifies immediately that $h$ is a cocycle. Let

$$
\phi: E^{(q)} \times E \rightarrow\left(E \times E^{(q)}\right)^{F}=E^{(q)} \times E
$$

be the identity morphism;

$$
\phi\left(h_{F}(X)\right)=\{0\} \times E^{(q)}+E \times\left\{0^{\prime}\right\}=X^{F} .
$$

Corollary. $B$ is a $\boldsymbol{F}_{q}$-simple abelian variety and $Y$ is a curve of genus two, irreducible over $\boldsymbol{F}_{q}$.

Proof. $B$ has Weil numbers $\pm q^{1 / 2}$ and is therefore $\boldsymbol{F}_{q}$-isogenous to $A$, hence $\boldsymbol{F}_{q}$-simple. $Y$ is an $\boldsymbol{F}_{q}$-irreducible curve on $B$ because the action of $h_{F}$ on $E \times E^{(q)}$ interchanges the two components of $X$ and $Y$ is the quotient of $X$ by this action. $Y$ has genus two because $Y \times F_{q^{2}} \cong X$. 
Theorem 1. $B$ admits infinitely many distinct principal polarizations defined over $\boldsymbol{F}_{q}$.

Proof. For any $a \in$ Aut $B$, the group of $\boldsymbol{F}_{q}$-automorphisms of $B$, the $\boldsymbol{F}_{q}$-rational divisor $a^{-1}(Y)$ determines a principal polarization of $B$ ([4], p. 63). The group of automorphisms of a polarized abelian variety is finite ([3], Proposition 8, p. 194), so there are only finitely many $b \in$ Aut $B$ such that $\mathscr{C}\left(b^{-1}(Y)\right)=\mathscr{C}\left(a^{-1}(Y)\right)$. On the other hand, $B$ has Weil numbers $\pm q^{1 / 2}$ so Aut $B$ contains an element of infinite order and the theorem follows.

Theorem 2. For any $a \in \operatorname{Aut} B, a^{-1}(Y)$ is a curve of genus two, irreducible over $\boldsymbol{F}_{q} . \quad$ Let $b \in$ Aut $B$ such that $\mathscr{C}\left(a^{-1}(Y)\right) \neq \mathscr{C}\left(b^{-1}(Y)\right)$, then $a^{-1}(Y)$ and $b^{-1}(Y)$ are geometrically non-isomorphic.

Proof. $\quad a^{-1}(Y) \times F_{q^{2}}=a^{-1}(X)=a^{-1}\left(E \times\left\{0^{\prime}\right\}\right)+a^{-1}\left(\{0\} \times E^{(q)}\right) . \quad E \times\left\{0^{\prime}\right\}$ and $\{0\} \times E^{(q)}$ are abelian subvarieties of $B$ so the two components of $a^{-1}(X)$ are abelian subvarieties of $B \times \boldsymbol{F}_{q^{2}}$. However, $B$ is a $\boldsymbol{F}_{q}$-simple abelian variety so neither of these components can be defined over $\boldsymbol{F}_{q}$. Hence $a^{-1}(Y)$ is $\boldsymbol{F}_{q}$-irreducible and is clearly of genus two. The second assertion follows immediately from Theorem 1 of [2].

$\S 2$.

Let $B\left(\boldsymbol{F}_{q^{m}}\right)$ denote the group of $\boldsymbol{F}_{q^{m}}$-rational points of $B$. Each $a \in$ Aut $B$ determines an isomorphism $a: B\left(\boldsymbol{F}_{q^{m}}\right) \rightarrow B\left(\boldsymbol{F}_{q^{m}}\right)$ for each $m$. Hence the singular curves $Y$ and $a(Y)$ have the same zeta function. $Y$ has only one $\boldsymbol{F}_{q}$-rational point, the one point of $X$ which is fixed under the action of $h_{F}$. This is the identity of $B$ and is the unique singular point of $Y$. This same point is the unique singular point of $a(Y)$.

Let $\tilde{Y}$ be the $\boldsymbol{F}_{q}$-normalization of $Y$ and $p: \tilde{Y} \rightarrow Y$ be the canonical projection. $\tilde{Y}$ is a complete, non-singular curve of genus two defined and irreducible over $\boldsymbol{F}_{q} . \quad \tilde{Y}$ is geometrically disconnected because $Y \times \boldsymbol{F}_{q^{2}}$ $=E \times\left\{0^{\prime}\right\}+\{0\} \times E^{(q)}$, so $\tilde{Y} \times F_{q^{2}}=E \times\left\{0^{\prime}\right\} \Perp\{0\} \times E^{(q)} . \quad \tilde{Y}$ can be recovered from $\tilde{Y} \times \boldsymbol{F}_{q^{2}}$ by a descent similar to the one used in the proof of the proposition. The action of $G$ on $\tilde{Y} \times \boldsymbol{F}_{q^{2}}$ has no fixed points so $\tilde{Y}$ has no $\boldsymbol{F}_{q}$-rational points; the singular point of $Y$ blows up to two $\boldsymbol{F}_{q^{2}}$ rational points on $\tilde{Y}$. Let $\zeta_{Y}$ denote the zeta function of $Y$. Then $\zeta_{\tilde{Y}}(T)$ $=\zeta_{Y}(T)(1-T) /\left(1-T^{2}\right)$. 
$E$ has Weil number $q$, so $\zeta_{E}(T)=(q T-1)^{2} /(1-T)\left(1-q^{2} T\right)$. Since $\tilde{Y}$ has no $F_{q^{r}}$-rational point for odd $r, \zeta_{\tilde{Y}}(T)=\zeta_{E}\left(T^{2}\right)$. Finally, $\zeta_{Y}(T)$ $=\left(q T^{2}-1\right)^{2} /(1+T)\left(1-T^{2}\right)\left(1-q^{2} T^{2}\right)$.

\section{REFERENCES}

[1] Honda, T., Isogeny classes of abelian varieties over finite fields, J. Math. Soc. Japan 20 (1968), 83-95.

[2] Hoyt, W. L., On products and algebraic families of Jacobian varieties, Ann. of Math. 77 (1963), 415-423.

[ 3 ] Lang, S., Abelian Varieties, Interscience, New York, 1959.

[ 4 ] Mumford, D., Abelian Varieties, Oxford University Press, London, 1970.

[ 5 ] Tate, J., Classes d'isogénie des variétés abéliennes sur un corps fini, Sem. Bourbaki $21(1968 / 69)$, no. 352.

[6] Tate, J., Endomorphisms of abelian varieties over finite fields, Invent. Math. 2 (1966), 134-144.

[ 7 ] Waterhouse, W., Abelian varieties over finite fields, Ann. scient. Éc. Norm. Sup. 4, t. 2 (1969), 521-560.

[ 8 ] Waterhouse W. and Milne, J. S., Abelian varieties over finite fields, in Proc. Symp. Pure Math. XX, American Mathematical Society, Providence, (1971), 53-64.

Pontificia Universidade Católica

do Rio de Janeiro 